\title{
Improving the safety and efficiency of outpatient lumbar puncture service
}

\author{
Mark Sweeney, Adam Al-Diwani, Robert Hadden
}

Kings College Hospital

\begin{abstract}
Lumbar puncture (LP) is a commonly performed procedure in diagnosis and management of neurological conditions. LP is generally safe, however there are a number of potentially serious complications, including epidural haematoma and cerebral herniation. The risks of these should be considered and minimised prior to undertaking LP.
\end{abstract}

Our regional neuroscience centre provides an outpatient LP service for patients throughout southeast England. Referrals from distant hospitals meant there was frequently no access to important clinical information, including indication for LP, past medical history, or medication history until the day of the procedure, and no access to results of investigations such as coagulation profile, platelet count, or intracranial imaging. Furthermore, there was limited capacity or time available in the day ward to perform these tests prior to LP. As a result, patients were either having LPs cancelled on the day of the procedure, were delayed by several hours on the day of the procedure for investigations, or were subject to the risk of having the LP performed without the knowledge of these key safety indicators.

To address this issue we implemented an LP safety checklist to be completed by referring neurologists, providing details of the patient's medical history and results of investigations performed locally. In doing this, we increased the proportion of patients with an available platelet count prior to LP from $25 \%$ to $89 \%$, and available coagulation profile from $18 \%$ to $82 \%$. In addition, we saw a qualitative increase in the confidence of junior doctors in the safety of the LP clinic, as measured by a survey taken before and after the implementation of this system.

This simple intervention made a rapid and remarkable difference to the safety and efficiency of this outpatient LP clinic. We would encourage other units to adopt this approach to address similar problems in a variety of outpatient settings.

\section{Problem}

Our regional neuroscience centre provides an outpatient lumbar puncture (LP) service for patients throughout southeast London, East Sussex, and Kent. Neurology consultants from district general hospitals (DGH) in this region refer patients to have LPs performed as a day case procedure. However, as these patients come from distant hospitals, we do not have access to their medical records, including relevant past medical history and current medication. In addition, the clinician performing the procedure does not have access to the results of key investigations performed in the referring hospital, such as platelet count, coagulation profile, and intracranial imaging. The clinic was under significant time pressure, and there was often insufficient time to perform such investigations on the day of the procedure, and as a result patients would either be cancelled and rearranged, or the procedure would be performed without knowledge of these key safety parameters. Here we describe the introduction of a safety checklist to be completed by the referring neurologist at the time of referral, to ensure these details are communicated from the referring neurologist, and are available to the clinician performing the procedure prior to the patient arriving for LP.

\section{Background}

Lumbar puncture is a commonly performed procedure in both inpatient and outpatient settings in elective and emergency circumstances, for diagnostic and occasionally therapeutic purposes.[1] Although LP is a relatively safe procedure, it carries a number of well described risks that must be considered and minimised to prevent potentially serious complications. These include post LP headache, infection, bleeding, cerebral herniation, radicular pain or numbness, late development of epidermoid tumours, and back pain.[1] The prevalence of thrombocytopenia or an abnormal coagulation profile in the general adult population is reported to be up to $17 \%$ in some studies, however the prevalence of clinically significant abnormalities is approximately $5 \%$.[2] In the setting of abnormal clotting, a lumbar epidural haematoma can develop, which causes compression of the spinal cord or cauda equina syndrome. Brainstem herniation ("coning") is potentially fatal. In particular, the risks of bleeding and brainstem herniation, the two most serious complications of LP, can be easily minimised by respectively: a) ensuring haemostatic parameters such as platelets and coagulation profile are normal prior to undertaking the procedure, $[3,4]$ and $b$ ) assessing for intracranial mass or raised intracranial pressure (ICP) either clinically or radiologically.[5]

Neurology services frequently perform elective LPs for diagnosis of neuroinflammatory or neurodegenerative conditions, [6, 7] and diagnosis and treatment of idiopathic intracranial hypertension (IIH).[8] In our centre, most outpatient LPs are performed by core medical trainees (CMTs) rotating through neurology, providing a valuable opportunity to perfect technical skills in addition to service provision. CMTs raised safety concerns due to the lack of availability of clinical information relating to risk of bleeding or 
cerebral mass lesion.

Given the lack of access to hospital records from distant referring centres, a judgement frequently had to be made as to the merits of repeating blood tests and/or computed tomography (CT) head scan, versus proceeding based upon clinical assessment of risk of bleeding and raised ICP alone. This decision making process was further complicated by the time pressured nature of the clinic: the unit was staffed until 1700 , and there were usually four patients booked for LP in the afternoon. Many of the referrals were technically difficult due to anxiety, cognitive and/or psychiatric disturbance, or obesity. Therefore, if the CMT doctor felt that further investigations were necessary in order to safely proceed, it was rarely possible to arrange these investigations and satisfy the appropriate safety checks before the unit closed. This caused cancellations and rearranged appointments, leading to patient frustration due to both the significant inconvenience of travelling long distances, and a delay in confirming or excluding anxiety provoking diagnoses such as multiple sclerosis. These factors often forced the doctor to do the LP without investigations, making only a clinical risk assessment of probability of haemostasis abnormalities or raised ICP. While this is not entirely unreasonable practice, we noted several near misses and themes that showed the risks to patient safety were not acceptable.

For example, on one occasion an LP was performed on an asymptomatic low risk patient without knowledge of their coagulation profile or platelet count. A full blood count was taken following the procedure for other reasons, and demonstrated a low platelet count of $54 \times 10^{\wedge} \mathrm{g} / \mathrm{L}$. Fortunately the patient had no complications from the procedure and no excess bleeding, but it could very easily have been different.

Another important patient category were those taking antithrombotic medication, for which there are specific guidelines for stopping prior to LP: namely, LP is generally considered unsafe when taking antithrombotics other than aspirin or dipyridamole monotherapy.[1] Patients had often not been given instructions on stopping antiplatelet medication prior to the procedure, leading to wasted journeys and appointment slots. Additionally, inexperience with new drugs such as the novel oral anticoagulants caused further confusion.

Furthermore, patients attending for LP with idiopathic intracranial hypertension commonly had symptoms of raised intracranial pressure and papilloedema, but without access to previous imaging results, it was necessary to arrange for obtaining the results of the scan on the day, or alternatively perform a new CT head scan. This led to the undesirable choice between a delay of some hours and unnecessary radiation exposure, or a cancelled and rearranged appointment.

It was clear to us that both efficient use of hospital resources, and patient time and safety were being unnecessarily compromised by a system failure, and that there was a pressing need to introduce a practical solution.

\section{Baseline measurement}

In order to assess the magnitude of this problem, we collected retrospective data over a two month period beginning 1 January 2015. Patients were identified from the admission office booking list, and we retrospectively reviewed the electronic patient notes of all patients having elective outpatient LPs in the neurology department at Kings College Hospital. This included the referral letter for LP, any blood tests performed in our hospital, and the notes made on the day by the doctor performing the procedure. We excluded any patients who failed to attend their appointment or were cancelled prior to the day of the procedure. We noted the number of patients who had a documented platelet count and documented coagulation testing available prior to the procedure. We used the international normalised ratio (INR) as the measure of clotting, as this was the most commonly available result. We also recorded the number of patients that had intracranial imaging results available, or documentation from the referring consultant neurologist stating this was not required because of low risk of raised ICP. We looked at the case mix, the number of patients who had procedures cancelled, and the reasons for these cancellations.

As shown in table 1, there were 40 cases in this two month period, and the majority of these $(63 \%)$ were referred for investigation of possible neuroinflammatory conditions such as multiple sclerosis, followed by idiopathic intracranial hypertension (18\%), and neurodegenerative conditions such as dementia (13\%).

Only $25 \%$ (10 of 40 ) of cases had a documented platelet count, and only $18 \%$ (7 of 40 ) had documented INR within six months prior to the LP being undertaken. The percentage of patients who had documented intracranial imaging was higher, at $75 \%$ (30 of 40 ).

Surprisingly, given the number of patients for whom there was no documented platelet count or coagulation profile, there was only one cancellation, which was due to the patient failing to stop antithrombotic medication. This suggested many LPs were being performed in suboptimal safety conditions, in order to prevent patients having to cancel and reattend. In addition, a number of procedures were performed after hours, due to the delay in receiving repeated blood test results, meaning the unit had to stay open and be staffed late, with significant delays to patients.

Furthermore, we conducted a small survey of the junior doctors performing the procedure, and found that $75 \%$ (3 out of 4 ) felt there were definite or major safety concerns with the patients referred for LP; however, as the small number of cancellations suggests, the LPs were being performed despite these concerns.

See supplementary file: ds6849.pdf - "Safety checklist"

\section{Design}

It was clear that in order to improve this service, we needed to ensure that results and extra information from the referring hospitals were available to the junior doctor prior to performing the procedure.

Initially, we attempted to improve the availability of this information 


\section{BMJ Quality Improvement Reports}

by arranging for the neurology CMTs to review the list of patients due to attend for LP that week on the Monday morning. If results were not available, the CMT would then be asked to call either the patient's GP surgery or their DGH to obtain the results of haemostatic tests and brain imaging, along with their medical history and medication list. Unfortunately this approach was particularly time consuming, as long periods of time had to be spent on the telephone to obtain these results, and GP surgeries often asked that requests for this information be faxed to them, adding further time delays to the process. Furthermore, patients on antithrombotic medication would still have had their appointments cancelled, as it would be too late to stop the medication in time for the procedure. Carrying out these checks in addition to ward work proved to be impractical.

We felt a much safer and more efficient approach would be to ask the referring neurologist to provide some simple details in the form of an LP safety checklist, which could be reviewed by the neurology CMTs prior to the patient being invited to attend for the procedure. Therefore, in consultation with neurology consultants both locally and in the referring hospitals, we developed a safety checklist which was to be completed by the referring neurologist (see supplement

1). Our aim was for the checklist to be short enough to be completed in less than one minute, while including important details about the patient and previous investigations undertaken at their local hospital, in order to minimise problems which arose on the day of the procedure.

The information requested on this safety checklist included:

- Indication for LP - Confirmation that platelet count and coagulation profile were
normal

- Confirmation that recent intracranial imaging showed no contraindication to LP, or an option to indicate that the patient was low risk of raised ICP and therefore did not require brain imaging

- Any medical history suggesting increased risk of bleeding

- Use of antithrombotic medication, and guidelines on how long each medication should be stopped prior to LP (provided by Dr Julia Czuprynska, consultant haematologist at King's College Hospital after consultation with her department)

- An option for the patient to be invited to attend the clinic three hours early to have blood tests, ensuring that the results would be available before the LP.

\section{Strategy}

When neurologists referred patients for an outpatient LP they were asked to complete the safety checklist, which was then reviewed by the neurology junior doctors prior to the patient being booked on the elective LP list. We included all patients booked for LP in the analysis whether or not they had a completed checklist, and excluded patients who did not attend their appointment or who were cancelled in advance.

We planned to analyse if there were improvements in availability of the three key safety parameters: coagulation profile, platelet count, and intracranial pressure assessment. We also hoped to see a reduction in the number of cancellations and the number of patients arriving who had failed to stop their antithrombotic medication. We used a chi squared test to determine if there was a significant difference before and after the implementation of the safety checklist.

\section{Results}

PDSA cycle 1: After implementing the system for two months we reanalysed the safety parameters (figure 1). In the two month period beginning 1 April 2015 there were 48 patients who attended for LP, with a similar mix of cases to the two months from the preimplementation retrospective study (table 1).

Following implementation of the safety checklist, there were clinically and statistically significant improvements: platelet count was available prior to LP in $71 \%$ (34 of $48, p<0.01$ ) of patients, and the INR was available in $58 \%$ ( 28 of $48, p<0.01$ ) of patients. The proportion of patients with intracranial imaging remained similar to the pre-implementation of the safety checklist $(77 \%, 37$ of 45$)$, however a further five patients had a statement on the checklist from the referring neurologist that intracranial imaging was not required. Overall, this resulted in a small non-significant improvement in intracranial pressure assessments to $88 \%$ (42 of 48). This change was also reflected in a repeat survey of the confidence of the junior doctors performing the LPs, as $100 \%$ (4 out of 4) responded that they had no safety concerns, or only minor safety concerns about patients referred for LP after the introduction of the safety checklist.

With regard to cancelled procedures, there was only one procedure which was cancelled on the day, and this was due to their blood results being unavailable; this patient had not been referred with a checklist, highlighting the utility of the checklist. The two patients who were taking antithrombotic medication had stopped this appropriately prior to the procedure, and there were therefore no cancelled procedures due to antithrombotic medication use.

We analysed the reasons for the large number of patients who continued to arrive for LPs without blood results. We found that a large proportion of these were due to the patient being booked for the procedure without a safety checklist. In total $25 \%$ (12 of 48 ) of patients arrived for LP without safety checklists.

The suboptimal uptake of the safety checklist was deemed to be due to a variety of reasons, including a lack of awareness of the checklist from referring consultants, patients who had booked appointments before the introduction of the checklist, and the fact that completing a paper form was time consuming and not user friendly. A further challenge we encountered at this point was the changeover of junior doctors.

PDSA cycle 2: We created an electronic checklist (supplement 1) 
that could be completed on a computer and emailed to the administrative team. In addition, the checklist was discussed at the regional consultant meeting to raise awareness of the new system, and the importance this provided in the service running smoothly.

We made a concerted effort to hand over this system to the incoming junior doctors, and ensured the process was well understood and would continue to be used effectively. Following these changes we repeated the data collection for a further two month period beginning on 1 June 2015, and found there had been significant further improvements. There were 45 patients attending for LP in this period, and on arriving for LP $89 \%$ ( 40 of $45, p<0.05$ ) had a documented platelet count, and $82 \%$ ( 37 of $45, \mathrm{p}<0.05$ ) had a documented INR. In addition, the percentage of patients with available intracranial imaging improved to $91 \%$ (41 of 45 ), and a further three patients were documented as low risk of raised ICP by their consultant neurologist, resulting in an overall significant improvement to $98 \%$ ( 44 of $45, p<0.01$ ) when compared to the prechecklist results. In this period there were three cancellations: two of these were due to the MRI scan taken on the day of the planned LP showing a contraindication to LP, and one was cancelled as there was no completed safety checklist and no blood tests available. The two patients who attended taking antithrombotic medication had appropriately stopped this prior to LP, allowing the procedure to be carried out.

Although not all patients were being booked with the safety checklist this had reduced markedly over time, with increasing awareness of the process and buy-in from the administrative team. In the second audit cycle there were only five patients (11\%) who attended without a completed checklist. On review, four of these patients were referred from within our trust and therefore had local investigation results available; however, we felt it remained important to continue encouraging this practice from all referring consultants, in particular to ensure that antithrombotic medications were not overlooked.

See supplementary file: ds6850.jpg - "Table 1 and figure 1"

\section{Lessons and limitations}

One difficulty we encountered in designing and implementing this project was the lack of available guidance regarding assessment of the risks of complications during lumbar puncture. In large scale analysis of the risks of LP, it is suggested that the most serious complications are rare in the absence of increased bleeding risk such as antithrombotic medication,[4] or risk factors for raised intracranial pressure such as papilloedema, impaired immunity, or recent onset of seizures.[1] Furthermore, these risks are considered only relative contraindications to LP. However, in the setting of elective outpatient diagnostic lumbar puncture with the potential for significant harm, we felt that with no clear guidance it was prudent to err on the side of caution with regards to risk factor assessment prior to lumbar puncture.

The involvement and assistance of the administrative team has been vital in ensuring the successful implementation of this project, and their continued support is even more vital in ensuring the sustainability of this intervention, as the junior doctors rotate regularly. Implementation of the safety checklist has required increased input and time from the administrative team, to send out the checklists and ensure that the results are checked and appointments booked in a timely fashion. We have found that the safety checklist has introduced some delays in the time taken for patients to be offered appointments for LP: previously, appointments were usually booked within 48 hours of receiving the referral, but after implementation of the checklist it can now take up to a week before an appointment can be booked. However, this has not resulted in any delay to the date of the LP. In addition, the new system has required extra time from CMTs to screen the safety checklists and act upon them as necessary; this typically amounted to less than one hour per week, however it has also resulted in time savings due to the more efficient running of the LP clinic, and avoiding the need to perform blood tests on the day. We have received feedback from patients that they find attending three hours early for blood tests frustrating, and therefore as much as possible we are aiming to avoid this by either using existing results from their local hospital, or asking their GP surgery to forward results prior to LP.

Despite these drawbacks, the benefits of the system were evident to the doctors performing this procedure as reflected in the survey of doctors and this has encouraged a change in culture regarding risk assessment prior to LP. We feel that the combination of this change in culture combined with the significant support from senior clinicians and the administrative team in the neurology department will serve to make these changes sustainable over the long term.

\section{Conclusion}

Prior to this project, there were significant efficiency and patient safety issues with the system in which elective LPs were being performed at our regional centre. This resulted in cancelled procedures or pressure to perform lumbar punctures in suboptimal safety conditions. By introducing a simple checklist to be completed by referring neurologists, we have greatly increased the number of patients who arrive with the appropriate investigations completed and available for the performing junior doctor. As exemplified qualitatively by the improvement in CMT doctors' confidence in the service, by implementing this project we have improved the safety culture, service efficiency, and have reduced the risks being taken by junior doctors to ensure a smooth running of the LP service. A simple intervention in a short period of time has dramatically improved patient safety and experience.

\section{References}

1. Johnson KS, Sexton DJ. Lumbar puncture: Technique, indications, contraindications, and complications in adults. http://www.uptodate.com/contents/lumbar-puncture-techniqu e-indications-contraindications-and-complications-in-adults

2. Houry S, Georgeac C, Hay JM, Fingerhut A, Boudet MJ. A prospective multicenter evaluation of preoperative hemostatic screening tests. The French Associations for Surgical Research. Am J Surg 1995;170(1):19-23.

3. Sinclair AJ, Carroll C, Davies B. Cauda equina syndrome 


\section{BMJ Quality Improvement Reports}

following a lumbar puncture. J Clin Neurosci

2009;16(5):714-6.

4. Pitkänen MT, Aromaa U, Cozanitis DA, Förster JG. Serious complications associated with spinal and epidural anaesthesia in Finland from 2000 to 2009. Acta Anaesthesiol Scand 2013;57(5):553-64.

5. van Crevel H, Hijdra A, de Gans J. Lumbar puncture and the risk of herniation: when should we first perform CT? J Neurol 2002;249(2):129-37.

6. Link $\mathrm{H}$, Huang YM. Oligoclonal bands in multiple sclerosis cerebrospinal fluid: an update on methodology and clinical usefulness. J Neuroimmunol 2006;180(1-2):17-28.

7. Blennow K, Dubois B, Fagan AM, Lewczuk P, de Leon MJ, Hampel $\mathrm{H}$. Clinical utility of cerebrospinal fluid biomarkers in the diagnosis of early Alzheimer's disease. Alzheimers Dement 2015;11(1):58-69.

8. Peng KP, Fuh JL, Wang SJ. High-pressure headaches: idiopathic intracranial hypertension and its mimics. Nat Rev Neurol 2012;8(12):700-10.

\section{Declaration of interests}

Nothing to declare.

\section{Acknowledgements}

We acknowledge the support of Ms June Luckraz (Neurology Admissions Coordinator).

\section{Ethical approval}

According to the Health Research Authority decision tool this work does not constitute a research study, and is classed as a service improvement project. We contacted the Research Ethics office in Kings College Hospital, who confirmed that ethical approval is not required for service improvement projects of this nature. 